\title{
Space-efficient and exact de Bruijn graph representation based on a Bloom filter
}

\author{
Rayan Chikhi ${ }^{*}$ and Guillaume Rizk²
}

\begin{abstract}
Background: The de Bruijn graph data structure is widely used in next-generation sequencing (NGS). Many programs, e.g. de novo assemblers, rely on in-memory representation of this graph. However, current techniques for representing the de Bruijn graph of a human genome require a large amount of memory ( $\geq 30 \mathrm{~GB}$ ).

Results: We propose a new encoding of the de Bruijn graph, which occupies an order of magnitude less space than current representations. The encoding is based on a Bloom filter, with an additional structure to remove critical false positives.

Conclusions: An assembly software implementing this structure, Minia, performed a complete de novo assembly of human genome short reads using $5.7 \mathrm{~GB}$ of memory in 23 hours.
\end{abstract}

Keywords: de novo assembly, de Bruijn graph, Bloom filter

\section{Background}

The de Bruijn graph of a set of DNA or RNA sequences is a data structure which plays an increasingly important role in next-generation sequencing applications. It was first introduced to perform de novo assembly of DNA sequences [1]. It has recently been used in a wider set of applications: de novo mRNA [2] and metagenome [3] assembly, genomic variants detection $[4,5]$ and de novo alternative splicing calling [6]. However, an important practical issue of this structure is its high memory footprint for large organisms. For instance, the straightforward encoding of the de Bruijn graph for the human genome ( $n \approx 2.4 \cdot 10^{9}, k$-mer size $k=27$ ) requires $15 \mathrm{~GB}$ ( $n \cdot k / 4$ bytes) of memory to store the nodes sequences alone. Graphs for much larger genomes and metagenomes cannot be constructed on a typical lab cluster, because of the prohibitive memory usage.

Recent research on de Bruijn graphs has been targeted on designing more lightweight data structures. Li et al. pioneered minimum-information de Bruijn graphs, by not recording read locations and paired-end information [7].

\footnotetext{
${ }^{*}$ Correspondence: chikhi@irisa.fr

${ }^{1}$ Computer Science department, ENS Cachan / IRISA / INRIA, Rennes 35042,

France

Full list of author information is available at the end of the article
}

Simpson et al. implemented a distributed de Bruijn graph to reduce the memory usage per node [8]. Conway and Bromage applied sparse bit array structures to store an implicit, immutable graph representation [9]. Targeted methods compute local assemblies around sequences of interest, using negligible memory, with greedy extensions [10] or portions of the de Bruijn graph [11]. Ye et al. recently showed that a graph roughly equivalent to the de Bruijn graph can be obtained by storing only one out of $g$ nodes $(10 \leq g \leq 25)[12]$.

Conway and Bromage observed that the selfinformation of the edges is a lower bound for exactly encoding the de Bruijn graph [9]:

$$
\left.\log _{2}\left(\begin{array}{c}
4^{k+1} \\
|E|
\end{array}\right)\right) \text { bits, }
$$

where $k+1$ is the length of the sequence that uniquely defines an edge, and $|E|$ is the number of edges. In this article, we will consider for simplicity that a de Bruijn graph is fully defined by its nodes. A similar lower bound can then be derived from the self-information of the nodes. For a human genome graph, the self-information of $|N| \approx 2.4 \cdot 10^{9}$ nodes is $\log _{2}\left(\left(\begin{array}{l}4^{k} \mid \\ |N|\end{array}\right)\right) \approx 6.8 \mathrm{~GB}$ for $k=27$, i.e. $\approx 24$ bits per node. 
A recent article [13] from Pell et al. introduced the probabilistic de Bruijn graph, which is a de Bruijn graph stored as a Bloom filter (described in the next section). It is shown that the graph can be encoded with as little as 4 bits per node. An important drawback of this representation is that the Bloom filter introduces false nodes and false branching. However, they observe that the global structure of the graph is approximately preserved, up to a certain false positive rate. Pell et al. did not perform assembly directly by traversing the probabilistic graph. Instead, they use the graph to partition the set of reads into smaller sets, which are then assembled in turns using a classical assembler. In the arXiv version of [13] (Dec 2011), it is unclear how much memory is required by the partitioning algorithm.

In this article, we focus on encoding an exact representation of the de Bruijn graph that efficiently implements the following operations:

1. For any node, enumerate its neighbors

2. Sequentially enumerate all the nodes

The first operation requires random access, hence is supported by a structure stored in memory. Specifically, we show in this article that a probabilistic de Bruijn graph can be used to perform the first operation exactly, by recording a set of troublesome false positives. The second operation can be done with sequential access to the list of nodes stored on disk. One highlight of our scheme is that the resulting memory usage is approximated by

$$
1.44 \log _{2}\left(\frac{16 k}{2.08}\right)+2.08 \text { bits } / k \text {-mer. }
$$

For the human genome example above and $k=27$, the size of the structure is $3.7 \mathrm{~GB}$, i.e. 13.2 bits per node. This is effectively below the self-information of the nodes. While this may appear surprising, this structure does not store the precise set of nodes in memory. In fact, compared to a classical de Bruijn graph, the membership of an arbitrary node cannot be efficiently answered by this representation. However, for the purpose of many applications (e.g. assembly), these membership queries are not needed.

We apply this representation to perform de novo assembly by traversing the graph. In our context, we refer by traversal to any algorithm which visits all the nodes of the graph exactly once (e.g. a depth-first search algorithm). Thus, a mechanism is needed to mark which nodes have already been visited. However, nodes of a probabilistic de Bruijn graph cannot store additional information. We show that recording only the visited complex nodes (those with in-degree or out-degree different than one) is a space-efficient solution. The combination of (i) the prob- abilistic de Bruijn graph along with the set of critical false positives, and (ii) the marking scheme, enables to perform very low-memory de novo assembly.

In the first Section, the notions of de Bruijn graphs and Bloom filters are formally defined. Section "Removing critical false positives" describes our scheme for exactly encoding the de Bruijn graph using a Bloom filter. Section "Additional marking structure for graph traversal" presents a solution for traversing our representation of the de Bruijn graph. Section "Results and discussion" presents two experimental results: (i) an evaluation of the usefulness of removing false positives and (ii) an assembly of a real human dataset using an implementation of the structure. A comparison is made with other recent assemblers based on de Bruijn graphs.

\section{de Bruijn graphs and Bloom filters}

The de Bruijn graph [1], for a set of strings $S$, is a directed graph. For simplicity, we adopt a node-centric definition. The nodes are all the $k$-length substrings (also called $k$-mers) of each string in $S$. An edge $s_{1} \rightarrow s_{2}$ is present if the $(k-1)$-length suffix of $s_{1}$ is also a prefix of $s_{2}$. Throughout this article, we will indifferently refer to a node and its $k$-mer sequence as the same object.

A more popular, edge-centric definition of de Bruijn graphs requires that edges reflect consecutive nodes. For $k /$-mer nodes, an edge $s_{1} \rightarrow s_{2}$ is present if there exists a $\left(k^{\prime}+1\right)$-mer in a string of $S$ containing $s_{1}$ as a prefix and $s_{2}$ as a suffix. The node-centric and edge-centric definitions are essentially equivalent when $k^{\prime}=k-1$ (although in the former, nodes have length $k$, and $k-1$ in the latter).

The Bloom filter [14] is a space efficient hash-based data structure, designed to test whether an element is in a set. It consists of a bit array of $m$ bits, initialized with zeros, and $h$ hash functions. To insert or test the membership of an element, $h$ hash values are computed, yielding $h$ array positions. The insert operation corresponds to setting all these positions to 1 . The membership operation returns yes if and only if all of the bits at these positions are 1. A no answer means the element is definitely not in the set. A yes answer indicates that the element may or may not be in the set. Hence, the Bloom filter has onesided errors. The probability of false positives increases with the number of elements inserted in the Bloom filter. When considering hash functions that yield equally likely positions in the bit array, and for large enough array size $m$ and number of inserted elements $n$, the false positive rate $\mathcal{F}$ is $[14]:$

$$
\mathcal{F} \approx\left(1-e^{-h n / m}\right)^{h}=\left(1-e^{-h / r}\right)^{h}
$$

where $r=m / n$ is the number of bits per element. For a fixed ratio $r$, minimizing Equation 1 yields the optimal number of hash functions $h \approx 0.7 r$, for which 
$\mathcal{F}$ is approximately $0.6185^{r}$. Solving Equation 1 for $m$, assuming that $h$ is the optimal number of hash function, yields $m \approx 1.44 \log _{2}\left(\frac{1}{\mathcal{F}}\right) n$.

The probabilistic de Bruijn graph is obtained by inserting all the nodes of a de Bruijn graph (i.e all $k$-mers) in a Bloom filter [13]. Edges are implicitly deduced by querying the Bloom filter for the membership of all possible extensions of a $k$-mer. Specifically, an extension of a $k$-mer $v$ is the concatenation of either (i) the $k-1$ suffix of $v$ with one of the four possible nucleotides, or (ii) one of the four nucleotides with the $k-1$ prefix of $v$.

The probabilistic de Bruijn graph holds an overapproximation of the original de Bruijn graph. Querying the Bloom filter for the existence of an arbitrary node may return a false positive answer (but never a false negative). This introduces false branching between original and false positive nodes.

\section{Removing critical false positives The cFP structure}

Our contribution is a mechanism that avoids false branching. Specifically, we propose to detect and store false positive elements which are responsible for false branching, in a separate structure. To this end, we introduce the $c F P$ structure of critical False Positives $k$-mers, implemented with a standard set allowing fast membership test. Each query to the Bloom filter is modified such that the yes answer is returned if and only if the Bloom filter answers $y e s$ and the element is not in $c F P$.

Naturally, if $c F P$ contained all the false positives elements, the benefits of using a Bloom filter for memory efficiency would be lost. The key observation is that the $k$-mers which will be queried when traversing the graph are not all possible $k$-mers. Let $\mathcal{S}$ be the set of true positive nodes, and $\mathcal{E}$ be the set of extensions of nodes from $\mathcal{S}$. Assuming we only traverse the graph by starting from a node in $\mathcal{S}$, false positives that do not belong to $\mathcal{E}$ will never be queried. Therefore, the set $c F P$ will be a subset of $\mathcal{E}$. Let $\mathcal{P}$ be the set of all elements of $\mathcal{E}$ for which the Bloom filter answers yes. The set of critical false positives $\boldsymbol{c F P}$ is then formally defined as $c F P=\mathcal{P} \backslash \mathcal{S}$.

Figure 1 shows a simple graph with the set $\mathcal{S}$ of correct nodes in regular circles and $c F P$ in dashed rectangles. The exact representation of the graph is therefore made of two data structures: the Bloom filter, and the set $c F P$ of critical false positives. Algorithm 1 describes how to construct $c F P$ using a fixed amount of memory. The set $\mathcal{P}$ is created on disk, from which $c F P$ is then gradually constructed by iteratively filtering $\mathcal{P}$ with partitions of $\mathcal{S}\left(\left(D_{i}\right)_{i \geq 0}\right)$ loaded in a hash-table. The sets $\mathcal{S}, \mathcal{P}$, and $\left(D_{i}\right)_{i \geq 0}$ are stored on the hard disk. The sets $\left(P_{i}\right)_{i \geq 0}$ reside in RAM, and are dimensioned to occupy as much space as the Bloom filter (which was freed at Step 4). Note that I/O to the disk are always sequential.

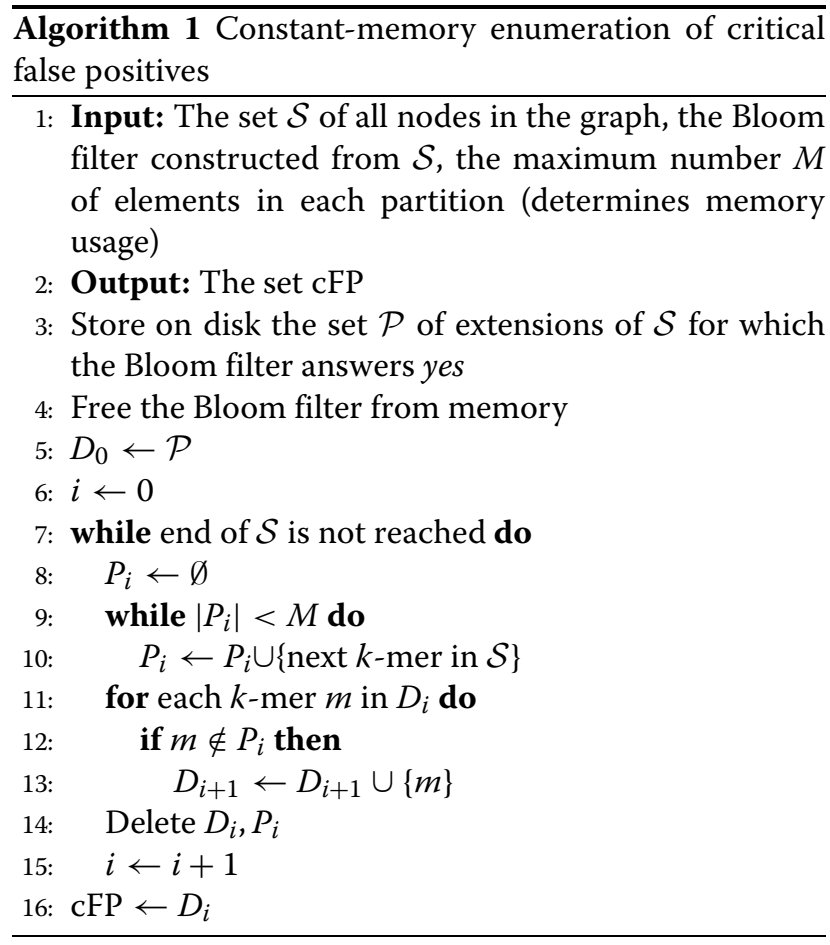

\section{Dimensioning the Bloom filter for minimal memory usage}

The set $c F P$ grows with the number of false positives. To optimize memory usage, a trade-off between the sizes of the Bloom filter and $c F P$ is studied here.

Using the same notations as in the definition of the Bloom filter, given that $n=|\mathcal{S}|$, the size of the filter $m$ and the false positive rate $\mathcal{F}$ are related through Equation 1. The expected size of $c F P$ is $8 n \cdot \mathcal{F}$, since each node only has eight possible extensions, which might be false positives. In the encoding of $c F P$, each $k$-mer occupies $2 \cdot k$ bits. Recall that for a given false positive rate $\mathcal{F}$, the expected optimal Bloom filter size is $1.44 n \log _{2}\left(\frac{1}{\mathcal{F}}\right)$. The total structure size is thus expected to be

$$
\underbrace{1.44 n \log _{2}\left(\frac{1}{\mathcal{F}}\right)}_{\text {Bloom filter }}+\underbrace{(16 \cdot \mathcal{F} n k)}_{c F P} \text { bits }
$$

The size is minimal for $\mathcal{F} \approx(16 k / 2.08)^{-1}$. Thus, the minimal number of bits required to store the Bloom filter and the set $c F P$ is approximately

$$
n \cdot\left(1.44 \log _{2}\left(\frac{16 k}{2.08}\right)+2.08\right) \text {. }
$$

For illustration, Figure 2-(a) shows the size of the structure for various Bloom filter sizes and $k=27$. For this value of $k$, the optimal size of the Bloom filter is 11.1 bits per $k$-mer, and the total structure occupies 13.2 bits per $k$-mer. Figure 2-(b) shows that $k$ has only a modest influ- 


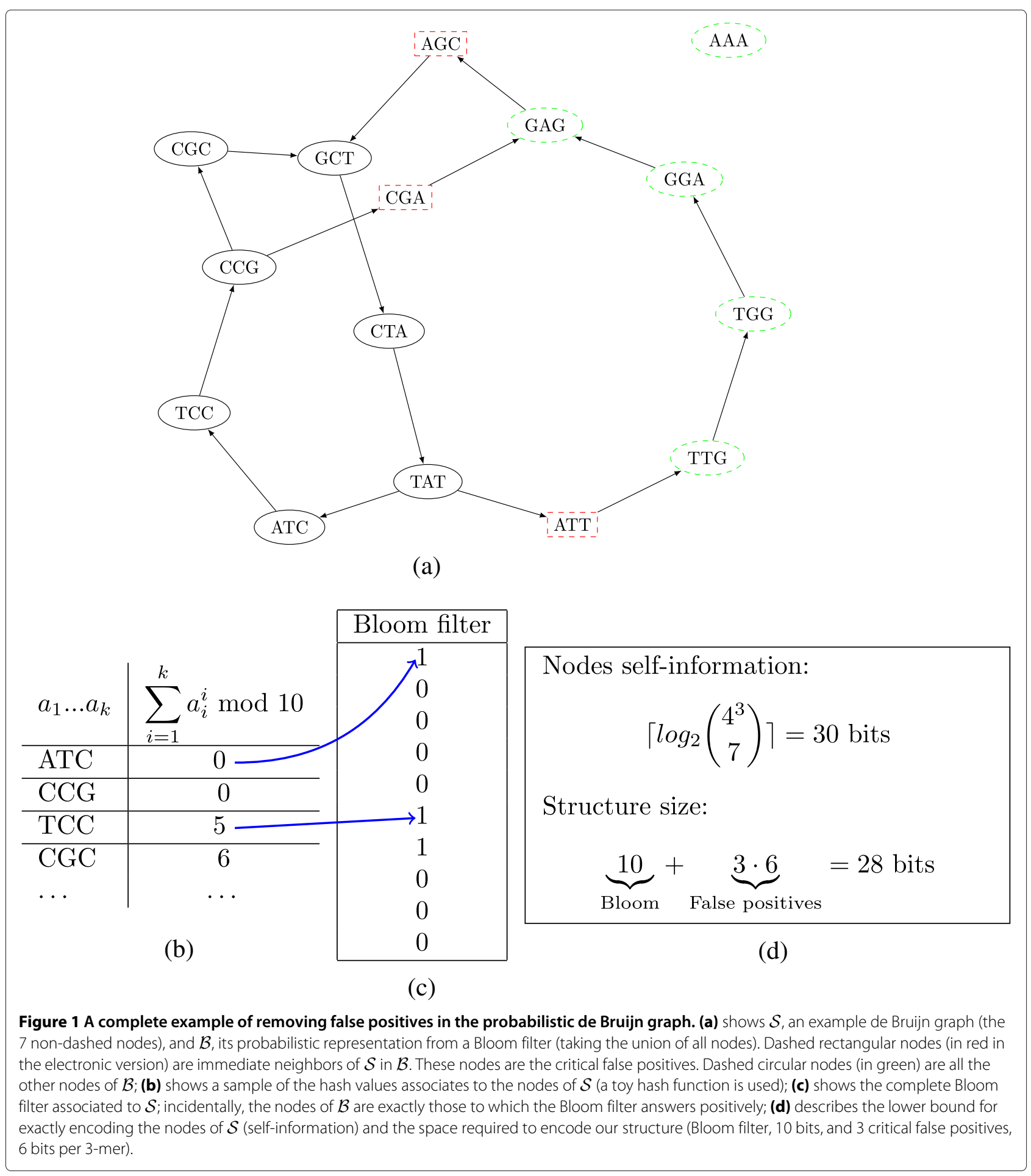

ence on the optimal structure size. Note that the size of the $c F P$ structure is in fact independent of $k$.

In comparison, a Bloom filter with virtually no critical false positives would require $\mathcal{F} \cdot 8 n<1$, i.e. $r>1.44$ $\log _{2}(8 n)$. For a human genome $\left(n=2.4 \cdot 10^{9}\right), r$ would be greater than 49.2, yielding a Bloom filter of size 13.7 GB.

\section{Additional marking structure for graph traversal}

Many NGS applications, e.g. de novo assembly of genomes [15] and transcriptomes [2], and de novo variant detection [6], rely on (i) simplifying and (ii) traversing the de Bruijn graph. However, the graph as represented in the previous section neither supports (i) simplifications (as it 


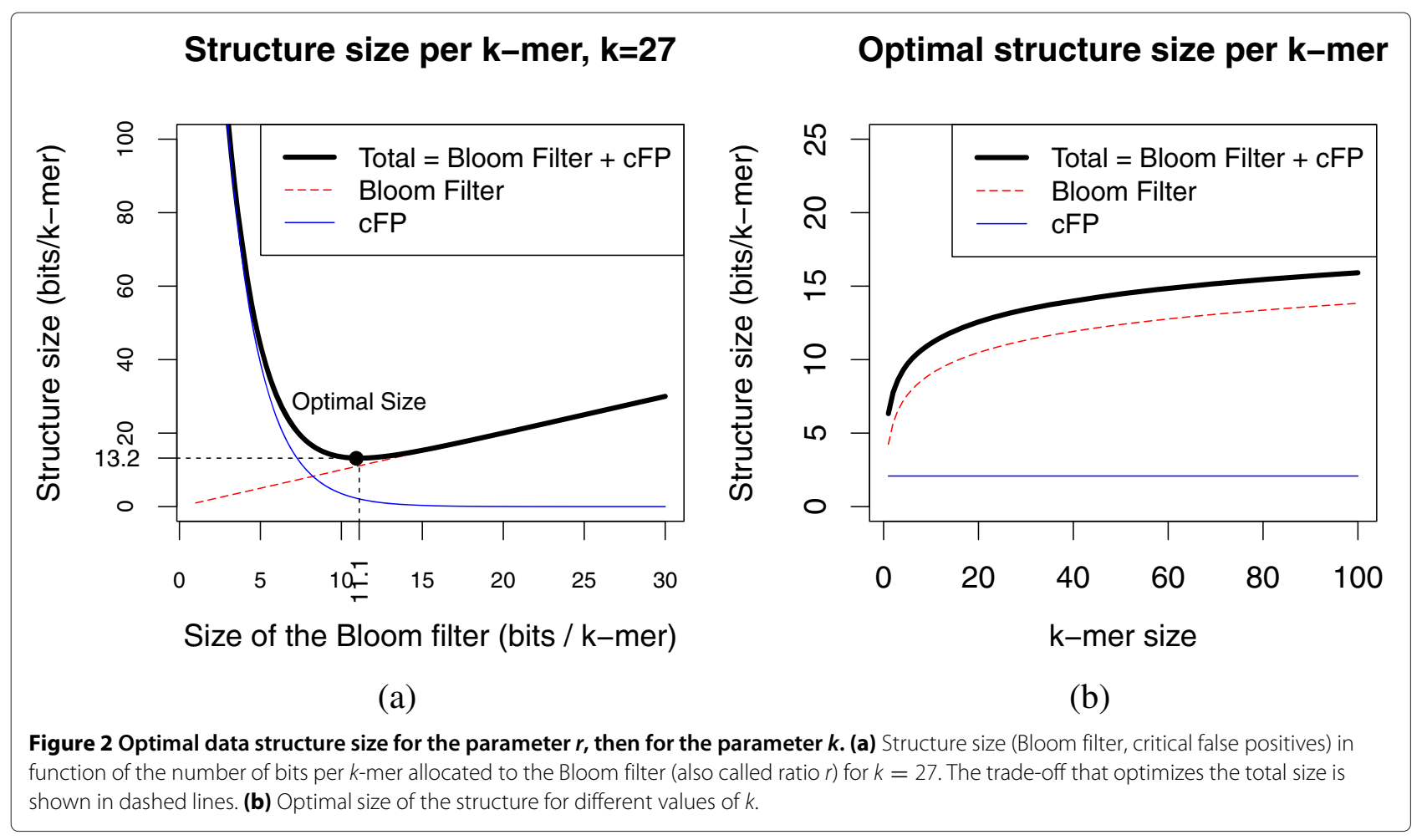

is immutable) nor (ii) traversals (as the Bloom filter cannot store an additional visited bit per node). To address the former issue, we argue that the simplification step can be avoided by designing a slightly more complex traversal procedure [16].

We introduce a novel, lightweight mechanism to record which portions of the graph have already been visited. The idea behind this mechanism is that not every node needs to be marked. Specifically, nodes that are inside simple paths (i.e nodes having an in-degree of 1 and an out-degree of 1) will either be all marked or all unmarked. We will refer to nodes having their in-degree or out-degree different to 1 as complex nodes. We propose to store marking information of complex nodes, by explicitly storing complex nodes in a separate hash table. In de Bruijn graphs of genomes, the complete set of nodes dwarfs the set of complex nodes, however the ratio depends on the genome complexity [17]. The memory usage of the marking structure is $n_{\mathrm{c}} C$, where $n_{\mathrm{c}}$ is the number of complex nodes in the graph and $C$ is the memory usage of each entry in the hash table $(C \approx 2 k+8)$.

\section{Implementation}

The de Bruijn graph structure described in this article was implemented in a new de novo assembly software: Minia ${ }^{\mathrm{a}}$. An important preliminary step is to retrieve the list of distinct $k$-mers that appear in the reads, i.e. true graph nodes.
To discard likely sequencing errors, only the $k$-mers which appear at least $d$ times are kept (solid $k$-mers). We experimentally set $d$ to 3 . Classical methods that retrieve solid $k$-mers are based on hash tables [18], and their memory usage scale linearly with the number of distinct $k$-mers. To deal with reverse-complementation, $k$-mers are identified to their reverse-complements.

\section{k-mer counting}

To avoid using more memory than the whole structure, we implemented a novel, constant-memory $k$-mer counting procedure. The multi-set of all $k$-mers present in the reads is partitioned and partitions are saved to disk. Then, each partition is separately loaded in memory in a temporary hash table. The $k$-mer counts are returned by traversing each hash table. Low-abundance $k$-mers are filtered. This approach permits to count all $k$-mers of a human genome dataset using only a fixed amount of memory and disk space. The algorithm is explicitly described and evaluated in another article [19].

\section{Graph traversal}

We implemented in Minia a graph traversal algorithm that constructs a set of contigs (gap-less sequences). The Bloom filter and the $c F P$ structure are used to determine neighbors of each node. The marking structure records already traversed nodes. A bounded-depth, boundedbreadth BFS algorithm (following Property 2 in [16]) 
is performed to traverse short, locally complex regions. Specifically, the traversal ignores tips (dead-end paths) shorter than $2 k+1$ nodes. It chooses a single path (consistently but arbitrarily), among all possible paths that traverse graph regions of breadth $\leq 20$, provided these regions end with a single node of depth $\leq 500$. These regions are assumed to be sequencing errors, short variants or short repetitions of length $\leq 500 \mathrm{bp}$. The breadth limit prevents combinatorial blowup. Note that pairedend reads information is not taken into account in this traversal. In a typical assembly pipeline (e.g. [8]), a separate program (scaffolder) can be used to link contigs using pairing information. Also, a gap-filling step (e.g. GapCloser in SOAPdenovo [7]) is typically used to fill the gaps between contigs in scaffolds.

\section{Results and discussion}

Throughout the Results section, we will refer to the N50 metric of an assembly (resp. NG50) as the longest contig size, such that half the assembly (resp. half the reference) is contained in contigs longer than this size.

\section{On the usefulness of removing critical false positives}

To test whether the combination of the Bloom filter and the $c F P$ structure offers an advantage over a plain probabilistic de Bruijn graph, we compared both structures in terms of memory usage and assembly consistency. We retrieved 20 million E. coli short reads from the Short Read Archive (SRX000429), and discarded pairing information. Using this dataset, we constructed the probabilistic de Bruijn graph, the $c F P$ structure, and marking structure, for various Bloom filter sizes (ranging from 5 to 19 bits per $k$-mer) and $k=23$ (yielding $4.7 \mathrm{M}$ solid $k$-mers).

We measured the memory usage of both structures. For each, we performed an assembly using Minia with exactly the same traversal procedure. The assemblies were compared to a reference assembly (using MUMmer), made with an exact graph. The percentage of nucleotides in contigs which aligned to the reference assembly was recorded.

Figure 3 shows that both the probabilistic de Bruijn graph and our structure have the same optimal Bloom filter size (11 bits per $k$-mer, total structure size of 13.82 bits and 13.62 per $k$-mer respectively). In the case of the probabilistic de Bruijn graph, the marking structure is prominent. This is because the graph has a significant amount of complex $k$-mers, most of them are linked to false positive nodes. For the graph equipped with the $c F P$ structure, the marking structure only records the actual complex nodes; it occupies consistently 0.49 bits per $k$-mer. Both structures have comparable memory usage.

However, Figure 3 shows that the probabilistic de Bruijn graph produces assemblies which strongly depend on the Bloom filter size. Even for large sizes, the probabilistic graph assemblies differ by more than $3 \mathrm{kbp}$ to the reference assembly. We observed that the majority of these differences were due to missing regions in the probabilistic graph assemblies. This is likely caused by extra branching, which shortens the lengths of some contigs (contigs shorter than $100 \mathrm{bp}$ are discarded).

Below $\approx 9$ bits per $k$-mer, probabilistic graph assemblies significantly deteriorate. This is consistent with another article [13], which observed that when the false positive
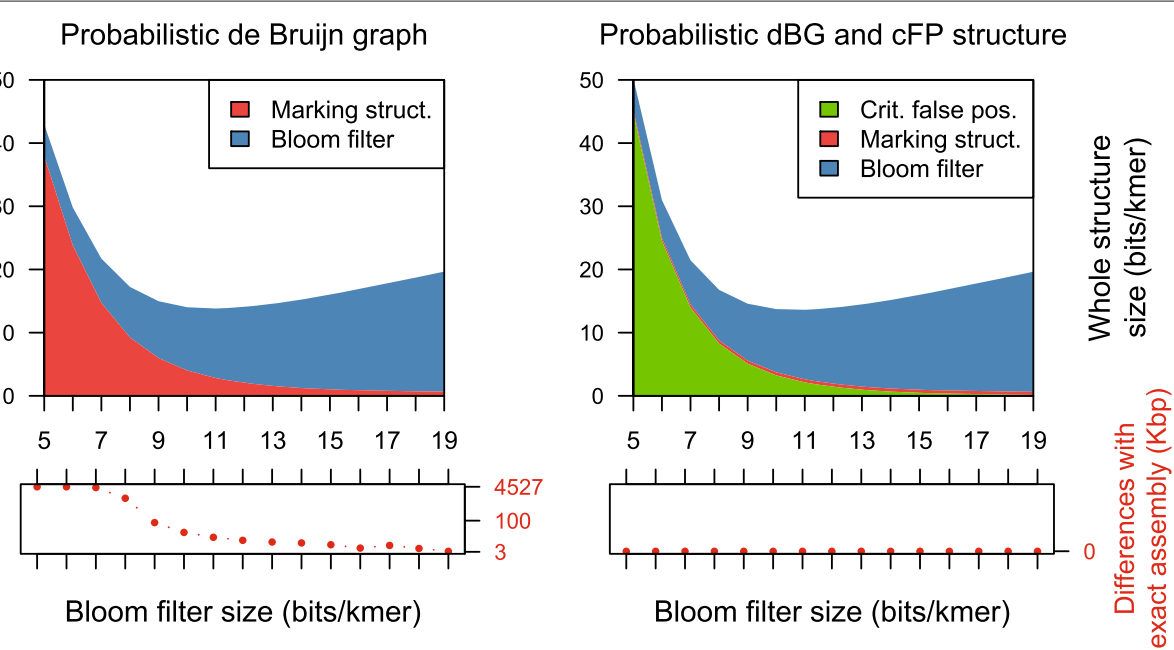

Figure 3 Data structure sizes for the probabilistic de Bruijn graph. Data structure sizes (Bloom filter, marking structure, and CFP if applicable) for the probabilistic de Bruijn graph with (top right) and without the CFP structure (top left), for an actual dataset (E. coli, $k=23$ ). All plots are in function of the number of bits per $k$-mer allocated to the Bloom filter. Additionally, the difference is shown (bottom left and bottom right) between a reference assembly made using an exact de Bruijn graph, and an assembly made with each structure. 
rate is over $18 \%$ (i.e., the Bloom filter occupies $\leq 4$ bits per $k$-mer), distant nodes in the original graph become connected in the probabilistic de Bruijn graph. To sum up, assemblies produced by the probabilistic de Bruijn graph are prone to randomness, while those produced by our structure are exact.

\section{de novo assembly Complete human genome}

We assembled a complete human genome (NA18507, SRA:SRX016231, $142.3 \mathrm{Gbp}$ of unfiltered reads of length $\approx 100 \mathrm{bp}$, representing $47 \mathrm{x}$ coverage) using Minia. After $k$-mer counting, $2,712,827,800$ solid $k$-mers $(d=3)$ were inserted in a Bloom filter dimensioned to 11.1 bits per solid $k$-mer. The $c F P$ structure contained $78,762,871$ $k$-mers, which were stored as a sorted list of 64 bits integers, representing 1.86 bits per solid $k$-mer. A total of $166,649,498$ complex $k$-mers ( $6 \%$ of the solid $k$-mers) were stored in the marking structure using 4.42 bits per solid $k$-mer (implementation uses $8\left\lceil\frac{k}{32}\right\rceil$ bytes per $k$-mer). Table 1 shows the time and memory usage required for each step in Minia.

We compared our results with assemblies reported by the authors of ABySS [8], SOAPdenovo [7], and the prototype assembler from Conway and Bromage [9]. Table 2 shows the results for four classical assembly quality metrics, and the time and peak memory usage of the compared programs. We note that Minia has the lowest memory usage (5.7 GB), seconded by the assembler from Conway and Bromage (32 GB). The wall-clock execution time of Minia ( $23 \mathrm{~h}$ ) is comparable to the other assemblers; note that it is the only single-threaded assembler. The N50 metric of our assembly $(1.2 \mathrm{kbp})$ is slightly above that of the other assemblies (seconded by SOAPdenovo, $0.9 \mathrm{kbp}$ ). All the programs except one assembled $2.1 \mathrm{Gbp}$ of sequences.

We furthermore assessed the accuracy of our assembly by aligning the contigs produced by Minia to the GRCh37 human reference using GASSST [20]. Out of the 2,090,828,207 nucleotides assembled, 1,978,520,767

Table 1 Details of steps implemented in Minia

\begin{tabular}{lll}
\hline Step & Time (h) & Memory (Gb) \\
\hline k-mer counting & 11.1 & Constant (set to 4.0) \\
Enumerating positive extensions & 2.8 & 3.6 (Bloom filter) \\
Constructing CFP & 2.9 & Constant (set to 4.0) \\
Assembly & 6.4 & $\begin{array}{l}5.7 \text { (Bloom f.+ CFP } \\
+ \text { mark. struct.) }\end{array}$ \\
\hline Overall & & 5.7 \\
\hline
\end{tabular}

Details of steps implemented in Minia, with wall-clock time and memory usage for the human genome assembly. For constant-memory steps, memory usage was automatically set to an estimation of the final memory size. In all steps, only one CPU core was used.
Table 2 de novo human genome (NA18507) assemblies

\begin{tabular}{lllll}
\hline Method & Minia & C. \& B. & ABySS & SOAPdenovo \\
\hline Value of $k$ chosen & 27 & 27 & 27 & 25 \\
\hline Number of contigs (M) & 3.49 & 7.69 & 4.35 & - \\
Longest contig (kbp) & 18.6 & 22.0 & 15.9 & - \\
Contig N50 (bp) & 1156 & 250 & 870 & 886 \\
Sum (Gbp) & 2.09 & 1.72 & 2.10 & 2.08 \\
\hline Nb of nodes/cores & $1 / 1$ & $1 / 8$ & $21 / 168$ & $1 / 16$ \\
Time (wall-clock, h) & 23 & 50 & 15 & 33 \\
$\begin{array}{l}\text { Memory (sum } \\
\text { of nodes, GB) }\end{array}$ & $\mathbf{5 . 7}$ & $\mathbf{3 2}$ & $\mathbf{3 3 6}$ & $\mathbf{1 4 0}$ \\
\hline
\end{tabular}

de novo human genome (NA18507) assemblies reported by our assembler (Minia), Conway and Bromage assembler [9], ABySS [8], and SOAPdenovo [7] Contigs shorter than $100 \mathrm{bp}$ were discarded. Assemblies were made without any pairing information.

nucleotides (94.6\%) were contained in contigs having a full-length alignment to the reference, with at least $98 \%$ sequence identity. For comparison, $94.2 \%$ of the contigs assembled by ABySS aligned full-length to the reference with $95 \%$ identity [8].

To evaluate another recent assembler, SparseAssembler [12], the authors assembled another dataset (NA12878), using much larger effective $k$ values. SparseAssembler stores an approximation of the de Bruijn graph, which can be compared to a classical graph for $k^{\prime}=k+g$, where $g$ is the sparseness factor. The reported assembly of the NA12878 individual by SparseAssembler $(k+g=56)$ has a N50 value of $2.1 \mathrm{kbp}$ and was assembled using 26 GB of memory, in a day. As an attempt to perform a fair comparison, we increased the value of $k$ from 27 to 51 for the assembly done in Table $2(k=56$ showed worse contiguity). The N50 obtained by Minia (2.0 kbp) was computed with respect to the size of SparseAssembler assembly. Minia assembled this dataset using 6.1 GB of memory in $27 \mathrm{~h}$, a $4.2 \times$ memory improvement compared to SparseAssembler.

\section{Chromosome 14 of the human genome}

In order to evaluate the quality of the results produced by Minia more accurately, we assembled the human chromosome 14 (88 Mbp ungapped) separately. The Illumina dataset is from the GAGE benchmark [21] (100 bp reads, all short paired-end libraries). Pairing information was not used in Minia. To establish a fair comparison with Minia, we selected two assemblies from GAGE (made with ABySS and Velvet, downloaded from the GAGE website) for which contigs were constructed without using pairing information. All the assemblies were done with a $k$-mer size of 31 , as chosen by the authors of GAGE. Additionnaly, we executed Minia with $k=47$, as this $k$-mer size was experimentally found to provide better results than $k=31$. 
QUAST v1.3 was executed with the --gage option to evaluate the quality of the contigs of each assembly. Table 3 shows several contiguity, coverage and quality metrics computed using the same reference genome and QUAST command-line for the three assemblies. The large misassemblies row shows the number of positions in the assembled contigs for which the left and right flanking sequences align either over $1 \mathrm{kbp}$ away from each other, or on different strands or chromosomes. The local misassemblies row shows the number of positions in the assembled contigs for which the left and right flanking sequences are distant from each other by $90 \mathrm{bp}$ to $1 \mathrm{kbp}$.

On this dataset, Minia with $k=31$ produced an assembly of lower contiguity and better accuracy to ABySS and Velvet. Minia with $k=47$ produced a better assembler over all metrics than the other assemblers run with $k=31$. Overall, the quality of assemblies produced with Minia can be considered satisfactory with respect to contigs made from state of the art assemblers. It should be noted that in the GAGE benchmark, other methods (e.g. SOAPdenovo, Allpaths, etc..) produced assemblies of much higher contiguity. These methods performed postprocessing steps (scaffolding and gap-filling), usually after completing an initial contigs construction phase with a classical de Bruijn graph.

\section{Conclusions}

This article introduces a new, space-efficient representation of the de Bruijn graph. The graph is implicitly encoded as a Bloom filter. A subset of false positives, those which introduce false branching from true positive nodes, are recorded in a separate structure. A new marking structure is introduced, in order for any traversal algorithm to mark which nodes have already been visited. The marking structure is also space-efficient, as it only stores information for a subset of $k$-mers. Combining the Bloom filter, the critical false positives structure and

Table 3 de novo human genome chromosome 14 assemblies

\begin{tabular}{lllll}
\hline Assembly & \multicolumn{2}{c}{ Minia } & ABySS & Velvet \\
\hline Value of $k$ chosen & 47 & 31 & 31 & 31 \\
\hline Number of contigs $(\mathrm{k})$ & 48.5 & 56.1 & 51.9 & 45.6 \\
Longest contig (kbp) & 28.9 & 26.5 & 30.0 & 27.9 \\
Contig NG50 (kbp) & 2.8 & 1.7 & 2.0 & 2.3 \\
\hline Large misassemblies & 11 & 3 & 20 & 385 \\
Local misassemblies & 25 & 27 & 158 & 867 \\
\hline Coverage (\%) & 92.4 & 81.9 & 82.2 & 84.4 \\
Unaligned contigs length (kbp) & 33.1 & 13.5 & 23.6 & 564.5 \\
\hline
\end{tabular}

de novo human genome chromosome 14 assemblies reported by our assembler (Minia), compared to Velvet and ABySS assemblies from the GAGE benchmark. QUAST was used to evaluate all three assemblers. the marking structure, we implemented a new memoryefficient method for de novo assembly (Minia).

To the best of our knowledge, Minia is the first method that can create contigs for a complete human genome on a desktop computer. Our method improves the memory usage of de Bruijn graphs by two orders of magnitude compared to ABySS and SOAPdenovo, and by roughly one order of magnitude compared to succinct and sparse de Bruijn graph constructions. Furthermore, the current implementation completes the assembly in 1 day using a single CPU thread.

De Bruijn graphs have more NGS applications than just de novo assembly. We plan to port our structure to replace the more expensive graph representations in two pipelines for reference-free alternative splicing detection, and SNP detection $[4,6]$. We wish to highlight three directions for improvement. First, some steps of Minia could be implemented in parallel, e.g. graph traversal. Second, a more succinct structure can be used to mark complex $k$-mers. Two candidates are Bloomier filters [22] and minimal perfect hashing.

Third, the set of critical false positives could be reduced, by exploiting the nature of the traversal algorithm used in Minia. The traversal ignores short tips, and in general, graph regions that are eventually unconnected. One could then define $n$-th order critical false positives $(n-c F P)$ as follows. An extension of a true positive graph node is a $n-c F P$ if and only if a breadth-first search from the true positive node, in the direction of the extension, has at least one node of depth $n+1$. In other words, false positive neighbors of the original graph which are part of tips, and generally local dead-end graph structures, will not be flagged as critical false positives. This is an extension of the method presented in this article which, in this notation, only detects 0 -th order critical false positives.

Simultaneously and independently of the present work, Bowe et al. [23] have proposed a de Bruijn graph representation using $4+o(1)$ bits per edge. Their data structure is more succinct than ours, and at the same time more complex and difficult to implement. Since their structure has not been fully implemented yet, it is not possible to compare the practical performance with Minia. However, this new advance is very interesting and raises the question of whether their structure is the most succinct way to represent a de Bruijn graph.

\section{Endnote}

${ }^{\text {a}}$ Source code available at http://minia.genouest.org/.

Competing interests

Both authors declare that they have no competing interests.

\section{Authors' contributions}

$R C$ and GR equally contributed to the data structure design, software and manuscript. Both authors read and approved the final manuscript. 
Acknowledgements

The authors are grateful to Dominique Lavenier for helpful discussions and advice, and to Aurélien Rizk for proof-reading the manuscript. This work benefited from the ANR grants associated with the MAPPI project (ANR-10-COSI-0004) and the GATB project (ANR-12-EMMA-0019).

\section{Author details}

'Computer Science department, ENS Cachan / IRISA / INRIA, Rennes 35042, France. ${ }^{2}$ Algorizk, Paris 75013, France.

Received: 19 December 2012 Accepted: 29 June 2013

Published: 16 September 2013

\section{References}

1. Idury RM, Waterman MS: A new algorithm for DNA sequence assembly. J Comput Biol 1995, 2(2):291-306.

2. Grabherr MG: Full-length transcriptome assembly from RNA-Seq data without a reference genome. Nat Biotech 2011, 29(7):644-652. [http://dx.doi.org/10.1038/nbt.1883]

3. Peng Y, Leung HCM, Yiu SM, Chin FYL: Meta-IDBA: a de Novo assembler for metagenomic data. Bioinformatics 2011, 27(13):i94-i101.

4. Peterlongo P, Schnel N, Pisanti N, Sagot MF, Lacroix V: Identifying SNPs without a reference genome by comparing raw reads. In String Processing and Information Retrieval. Berlin, Heidelberg: Springer; 2010:147-158.

5. Iqbal Z, Caccamo M, Turner I, Flicek P, McVean G: De novo assembly and genotyping of variants using colored de Bruijn graphs. Nat Genet 2012, 44:226-232.

6. Sacomoto G, Kielbassa J, Chikhi R, Uricaru R, Antoniou P, Sagot M, Peterlongo P, Lacroix V: KISSPLICE: de-novo calling alternative splicing events from RNA-seq data. BMC Bioinformatics 2012, 13(Suppl 6):S5. [http://www.biomedcentral.com/1471-2105/13/S6/S5]

7. Li R, Zhu H, Ruan J, Qian W, Fang X, Shi Z, Li Y, Li S, Shan G, Kristiansen K: De novo assembly of human genomes with massively parallel short read sequencing. Genome Res 2010, 20(2):265.

8. Simpson JT, Wong K, Jackman SD, Schein JE, Jones SJM, Birol I: ABySS: A parallel assembler for short read sequence data. Genome Res 2009, 19(6):1117-1123. [http://genome.cshlp.org/content/19/6/1117.abstract]

9. Conway TC, Bromage AJ: Succinct data structures for assembling large genomes. Bioinformatics 2011, 27(4):479.

10. Warren RL, Holt RA: Targeted assembly of short sequence reads. PloS One 2011, 6(5):e19816.

11. Peterlongo $P$, Chikhi R: Mapsembler, targeted and micro assembly of large NGS datasets on a desktop computer. BMC Bioinformatics 2012, 13:48.

12. Ye C, Ma Z, Cannon C, Pop M, Yu D: Exploiting sparseness in de novo genome assembly. BMC Bioinformatics 2012, 13(Suppl 6):S1. [http://www.biomedcentral.com/1471-2105/13/S6/S1]

13. Pell J, Hintze A, Canino-Koning R, Howe A, Tiedje JM, Brown CT: Scaling metagenome sequence assembly with probabilistic de Bruijn graphs. Arxiv preprint arXiv:1112.41932011.

14. Kirsch A, Mitzenmacher M: Less hashing, same performance: Building a better Bloom filter. Algorithms-ESA 2006, 4168:456-467.

15. Miller JR, Koren S, Sutton G: Assembly algorithms for next-generation sequencing data. Genomics 2010, 95(6):315-327.

16. Chikhi $R$, Lavenier $D$ : Localized genome assembly from reads to scaffolds: practical traversal of the paired string graph. Algo Bioinformatics 2011, 6833:39-48.

17. Kingsford C, Schatz MC, Pop M: Assembly complexity of prokaryotic genomes using short reads. BMC Bioinformatics 2010, 11:21.

18. Marçais $G$, Kingsford $C$ : A fast, lock-free approach for efficient parallel counting of occurrences of k-mers. Bioinformatics 2011, 27(6):764-770. [http://bioinformatics.oxfordjournals.org/content/27/6/764.abstract]

19. Rizk G, Lavenier D, Chikhi R: DSK: $\boldsymbol{k}$-mer counting with very low memory usage. Bioinformatics 2013, 29(5):652-653.

20. Rizk G, Lavenier D: GASSST: global alignment short sequence search tool. Bioinformatics 2010, 26(20):2534.

21. Salzberg SL, Phillippy AM, Zimin A, Puiu D, Magoc T, Koren S, Treangen TJ, Schatz MC, Delcher AL, Roberts M, Marçais G, Pop M, Yorke JA: GAGE: A critical evaluation of genome assemblies and assembly algorithms.
Genome Res 2012, 22(3):557-567. [http://genome.cshlp.org/content/22/ 3/557.abstract]

22. Chazelle B, Kilian J, Rubinfeld R, Tal A: The Bloomier filter: an efficient data structure for static support lookup tables. In Proceedings of the Fifteenth Annual ACM-SIAM Symposium on Discrete Algorithms. Philadelphia: SIAM; 2004:30-39.

23. Bowe A, Onodera T, Sadakane K, Shibuya T: Succinct de Bruijn Graphs. In Algorithms in Bioinformatics, Volume 7534 of Lecture Notes in Computer Science. Edited by Raphael B, Tang J. Berlin, Heidelberg: Springer; 2012:225-235. [http://dx.doi.org/10.1007/978-3-642-33122-0_18]

doi:10.1186/1748-7188-8-22

Cite this article as: Chikhi and Rizk: Space-efficient and exact de Bruijn graph representation based on a Bloom filter. Algorithms for Molecular Biology 2013 8:22.

\section{Submit your next manuscript to BioMed Central and take full advantage of:}

- Convenient online submission

- Thorough peer review

- No space constraints or color figure charges

- Immediate publication on acceptance

- Inclusion in PubMed, CAS, Scopus and Google Scholar

- Research which is freely available for redistribution
Biomed Central 